\title{
Impact of Tricuspid Annuloplasty on Postoperative Changes in The Right Ventricular Systolic and Diastolic Function: A Retrospective Cohort Study
}

\author{
Tomoki Sakata ${ }^{1}$, Kenji Mogi ${ }^{1}$, Manabu Sakurai ${ }^{2}$, Kengo Tani $^{1}$, Masafumi Hashimoto ${ }^{1}$, Yuki \\ Shiko $^{3}$, Yohei Kawasaki ${ }^{3}$, Goro Matsumiya ${ }^{3}$, and Yoshiharu Takahara ${ }^{1}$ \\ ${ }^{1}$ Funabashi Municipal Medical Center Heart and Vascular Institute \\ ${ }^{2}$ Funabashi Municipal Medical Center \\ ${ }^{3}$ Chiba University Hospital
}

April 27, 2020

\begin{abstract}
Objectives: To elucidate the impact of regulation of tricuspid regurgitation (TR) using tricuspid annuloplasty on postoperative changes in right ventricular (RV) systolic and diastolic functions. Methods: We enrolled 69 patients who underwent aortic or mitral valve surgery between July 2016 to March 2018 without recurrence. Patients with concomitant coronary artery bypass grafting or a history of previous cardiovascular surgery were excluded, remaining 45 patients enrolled. Patients were divided into 2 groups according to concomitant tricuspid annuloplasty ( $\mathrm{T}: \mathrm{n}=12$ vs non- $\mathrm{T}: \mathrm{n}=33$ ). RV global longitudinal strain (RVGLS), RV fractional area change (RVFAC), tricuspid annular plane systolic excursion (TAPSE) and early tricuspid inflow velocity/early diastolic tricuspid annular velocity ratio (tricuspid E/e') were assessed as functional indices at preoperative, postoperative and 1-year follow-up periods. Results: RVFAC deteriorated postoperatively but recovered at follow-up in group T, whereas that in group non-T showed gradual deterioration overtime. RVGLS and TAPSE showed similar temporary deterioration and recovery between groups. Tricuspid $\mathrm{E}$ in group $\mathrm{T}$ increased postoperatively and showed significant difference, which was kept until follow-up period. Tricuspid e' decreased postoperatively, and recovered slightly in both groups. As a result, postoperative RV diastolic function (tricuspid E/e') showed significant difference between groups. This difference was maintained until follow-up. Conclusions: RV systolic function deteriorated postoperatively, but there was a tendency to improve at follow-up regardless of tricuspid annuloplasty. RV diastolic function may potentially be impaired when TR was regulated by tricuspid annuloplasty.
\end{abstract}

\section{Hosted file}

Manuscript.doc available at https : //authorea.com/users/315753/articles/446030-impact-of-tricuspidannuloplasty-on-postoperative-changes-in-the-right-ventricular-systolic-and-diastolic-functiona-retrospective-cohort-study 

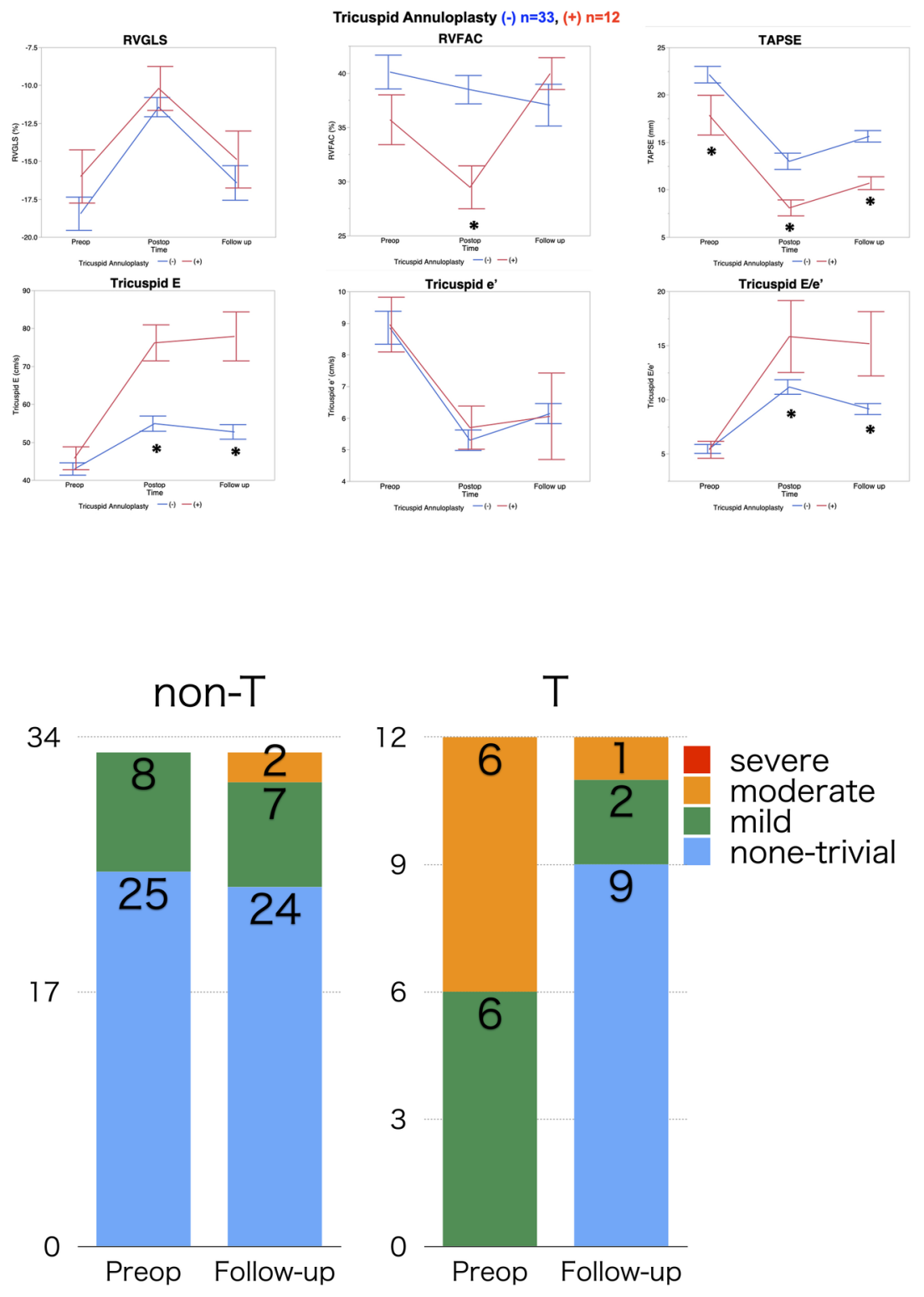

\section{Hosted file}

Table 1.doc available at https://authorea.com/users/315753/articles/446030-impact-of-tricuspidannuloplasty-on-postoperative-changes-in-the-right-ventricular-systolic-and-diastolic-functiona-retrospective-cohort-study

\section{Hosted file}

Table 2.doc available at https://authorea.com/users/315753/articles/446030-impact-of-tricuspidannuloplasty-on-postoperative-changes-in-the-right-ventricular-systolic-and-diastolic-functiona-retrospective-cohort-study 